\title{
Genotype-phenotype correlation and frequency of the 3199del6 cystic fibrosis mutation among I148T carriers: Results from a collaborative study
}

\author{
K. G. Monaghan ${ }^{1}$, W. E. Highsmith ${ }^{2}$, J. Amos ${ }^{3}$, V. M. Pratt ${ }^{4}$, B. Roa ${ }^{5}$, M. Friez ${ }^{6}$, L. L. Pike-Buchanan ${ }^{4}$, I. M. Buyse ${ }^{5}$,
} J. B. Redman ${ }^{7}$, C. M. Strom ${ }^{7}$, A. L. Young ${ }^{2}$, and W. Sun ${ }^{7}$

\begin{abstract}
Purpose: We expect that the mutation panel currently recommended for preconception/prenatal CF carrier screening will be modified as new information is learned regarding the phenotype associated with specific mutations and allele frequencies in various populations. One such example is the I148T mutation, originally described as a severe $\mathrm{CF}$ mutation. After implementation of CF population-based carrier screening, we learned that I148T exists as a complex allele with 3199del6 in patients with clinical CF, whereas asymptomatic compound heterozygotes for I148T and a second severe CF mutation were negative for 3199del6. Methods: We performed reflex testing for 3199del6 on 663 unrelated specimens, including I148T heterozygotes, compound heterozygotes, and a homozygous individual. Results: Less than $1 \%$ of $1148 \mathrm{~T}$ carriers were also positive for 3199del6. Excluding subjects tested because of a suspected or known CF diagnosis or positive family history, 0.6\% of I148T-positive individuals were also positive for 3199del6. We identified 1 I148T homozygote and 6 unrelated compound heterozygous individuals with I148T and a second CF variant (2 of whom also carried 3199del6). In addition, one fetus with echogenic bowel and one infertile male were heterozygous for I148T (3199del6 negative). Conclusions: Reflex testing for 3199del6 should be considered whenever I148T is identified. Reflex testing is of particular importance for any symptomatic patient or whenever one member of a couple carries a deleterious CF mutation and the other member is an I148T heterozygote. Further population data are required to determine if I148T, in the absence of 3199del6, is associated with mild or atypical CF or male infertility. Genet Med 2004:6(5):421-425.
\end{abstract}

Key Words: cystic fibrosis, mutation analysis, 3199del6, I148T

Over 1000 cystic fibrosis (CF) mutations have been identified; most are rare, having been detected in only one family. ${ }^{1}$ In 2001, the $\mathrm{ACMG}^{2}$ and $\mathrm{ACOG}^{3}$ developed a panethnic panel of 25 common mutations with a frequency $\geq 0.1 \%$ in the general population and recommended that all Caucasians who are either pregnant or considering pregnancy be screened. ACMG/ ACOG also recommend that screening be made available to individuals in lower risk ethnic groups. We expect that the mutation panel will be modified as new information arises regarding the phenotype associated with specific mutations and allele frequencies in various populations. The I148T mutation, which is currently included in the panel, was first reported in

\footnotetext{
From the ${ }^{1}$ Henry Ford Hospital, Detroit, Michigan; ${ }^{2}$ Mayo Medical Laboratories, Rochester, Minnesota; ${ }^{3}$ Specialty Laboratories, Santa Monica, California; ${ }^{4}$ Quest Diagnostics Nichols Institute, Chantilly, Virginia; ${ }^{5}$ Baylor College of Medicine, Houston, Texas; ${ }^{6}$ Greenwood Genetic Center, Greenwood, South Carolina; ${ }^{7}$ Quest Diagnostics Nichols Institute, San Juan Capistrano, California.

Kristin G. Monaghan, PhD, Department of Medical Genetics, CFP-4, Henry Ford Hospital, Detroit, MI 48202.

Received: March 8, 2004.

Accepted: May 13, 2004.
}

DOI: 10.1097/01.GIM.0000139507.20179.3A
1990 as a severe mutation ${ }^{4-7}$ and accounted for $9 \%$ of FrenchCanadian CF mutations. ${ }^{8}$ After implementation of CF population-based carrier screening, two studies noted a $>100$-fold increase in the frequency of I148T among individuals undergoing carrier screening compared to patients with clinical CF. ${ }^{9-10}$ Further studies revealed a second mutation, 3199del6, in-cis with I148T in affected patients, ${ }^{9,11}$ a finding previously reported in 1998. ${ }^{1}$ The 3199del6 mutation was not present in asymptomatic compound heterozygous individuals with I148T and a second, severe CF mutation, such as $\Delta$ F508. Initial studies reported that approximately $1.8 \%$ of I148T heterozygotes identified by carrier screening also have the 3199 del 6 mutation. ${ }^{9}$ Subsequent studies identified 3199del6 in $0.9 \%$ of I148T carriers and determined the frequency of 3199 del 6 in the general population to be $<0.1 \% .^{12}$ We have summarized the cases in which I148T was detected in our laboratories and report the frequency of 3199del6 among 663 I148T-positive individuals.

\section{MATERIALS AND METHODS}

\section{Patient population}

Reflex testing for 3199del6 was performed on 663 patients positive for I148T among the 7 participating laboratories be- 
tween 6/95 and 11/03. The ethnicity/races of these individuals included African American, Caucasian, Hispanic, Middle Eastern, and Asian. One hundred forty-three adult subjects were tested for the indication of carrier screen, and 505 had an unspecified indication, although most are presumed to have been referred for carrier screening. The indications for the remaining cases were as follows: suspected diagnosis of CF (7), family history of a CF mutation (2), male infertility (3), clinical diagnosis of CF (1), and fetal echogenic bowel (2).

Some of the individuals whose data are included in this article were also included in the recently published article by Buller et al. ${ }^{12}$ The previous study analyzed 3199 del6 by a different method (Promega ReadIT), whereas the analysis reported in this study was done by DNA sequencing. Some patients were tested by both methods and therefore included in both articles. Samples previously published were tested anonymously, so there is no way to determine which specific individuals were already reported, with the exception of Case 1 (Table 2), who is known to be included in the previously published study.

\section{I148T and 3199del6 mutation analysis}

\section{Henry Ford Hospital}

CF studies for I148T were performed by PCR amplification followed by either a laboratory-developed method involving heteroduplex analysis and RFLP with BsrI or oligonucleotide ligation assay (CF OLA v3.0) (Celera Diagnostics/Abbott Diagnostics/Applied Biosystems). Reflex testing for 3199del6 was performed on all individuals positive for I148T using a laboratory-developed PCR and polyacrylamide gel electrophoresis (PAGE) assay.

\section{Mayo Clinic}

CF studies were performed using either Confirmation Sensitive Gel Electrophoresis (CSGE) or the INNO-LiPA CFTR Assay System (Innogenetics). Reflex testing for 3199del6 was performed using a laboratory-developed PCR and heteroduplex assay.

\section{Specialty Laboratories}

CF studies were performed using the Linear Array CF Gold 1.0 assay (Roche Molecular Biochemicals). A laboratory-developed assay utilizing PCR and PAGE was used for the detection of 3199del6.

\section{Quest Diagnostics}

CF studies were performed by PCR amplification followed by oligonucleotide ligation assay (CF OLA v3.0) (Celera Diagnostics/Abbott Diagnostics/Applied Biosystems). Requested testing for 3199del6 was performed using a laboratory-developed direct sequence analysis of exon 17a of the CFTR gene. ${ }^{13}$

\section{Baylor College of Medicine}

CF direct mutation analysis was done using a matrix assisted laser desorption ionization-time of flight (MALDI-TOF) mass spectrometry platform (Sequenom), which includes the I148T and 3199del6 mutations.

\section{Greenwood Genetic Center}

CF studies for I148T were performed by PCR amplification followed by either laboratory-developed sequencing analysis or oligonucleotide ligation assay (CF OLA v3.0) (Celera Diagnostics/Abbott Diagnostics/Applied Biosystems). Reflex testing for 3199del6 was performed with a laboratory-developed sequencing assay.

\section{RESULTS}

A summary of the 3199del6- and/or I148T-positive patients identified is shown in Table 1. Data were collected on 662 un-

Table 1

Summary of 3199del6 and/or I148T chromosomes identified among 663 individuals undergoing CF DNA testing

\begin{tabular}{|c|c|c|c|}
\hline Indication for DNA testing & Race/Ethnicity & $\begin{array}{l}\mathrm{I} 148 \mathrm{~T} \\
\text { positive }\end{array}$ & $\begin{array}{l}\text { 3199del6 } \\
\text { positive }\end{array}$ \\
\hline Carrier screen & Caucasian & 71 & 0 \\
\hline Unspecified $^{a}$ & Caucasian & 128 & 0 \\
\hline Carrier screen & Hispanic & 8 & 0 \\
\hline Unspecified $^{a}$ & Hispanic & 14 & 1 \\
\hline Carrier screen & Middle Eastern & 7 & 0 \\
\hline Unspecified $^{a}$ & Middle Eastern & 6 & 0 \\
\hline Carrier screen & Other & 2 & 0 \\
\hline Carrier screen & Asian & 5 & 0 \\
\hline Unspecified $^{a}$ & Asian & $14^{b}$ & 0 \\
\hline Carrier screen & African American & 3 & 0 \\
\hline Carrier screen & Unspecified & 47 & 0 \\
\hline Unspecified $^{a}$ & Other & 3 & 0 \\
\hline Unspecified $^{a}$ & Unspecified & 341 & 3 \\
\hline Subtotal & & 649 & $4(0.6 \%)$ \\
\hline Fetus with echogenic bowel & Middle Eastern & 1 & 0 \\
\hline Fetus with echogenic bowel & Asian & 1 & 0 \\
\hline Male infertility & Caucasian & 1 & 0 \\
\hline Male infertility & Unspecified & 2 & 0 \\
\hline Rule-out CF & Unspecified & 6 & 0 \\
\hline Rule-out CF & African American & 1 & 0 \\
\hline Clinical CF & Caucasian & 1 & 1 \\
\hline Family history of CF mutation & Armenian & 1 & 1 \\
\hline Family history of a CF mutation & Caucasian & 1 & 0 \\
\hline Subtotal & & 15 & $2(13.3 \%$ \\
\hline Total & & 664 & $6(0.9 \%)$ \\
\hline
\end{tabular}

${ }^{a}$ The majority of persons undergoing CF DNA testing for an unspecified indication are presumably undergoing carrier screening.

${ }^{b} 12$ individuals were heterozygous for I148T, and 1 individual was I148T homozygous. 
Table 2

Summary of interesting cases

\begin{tabular}{|c|c|c|c|c|c|c|}
\hline Case no. & Sex & Race & Indication & Genotype & Poly T status & Clinical information \\
\hline 1 & Female & Asian & Carrier screening & $\mathrm{I}^{148 \mathrm{~T}^{a}} / \mathrm{I}_{148 \mathrm{~T}^{a 12}}$ & $9 \mathrm{~T} / 9 \mathrm{~T}$ & Asymptomatic \\
\hline 2 & Male & Not provided & Infertility & $\mathrm{I} 148 \mathrm{~T}^{a} / \Delta \mathrm{F} 508$ & Not determined & CBAVD \\
\hline 3 & Male & Not provided & Infertility & I148 $^{a}$ heterozygote & Negative for $5 \mathrm{~T}$ & Obstructive azoospermia \\
\hline 4 & Male & Caucasian & Infertility & $\mathrm{I} 148 \mathrm{~T}^{a} / \mathrm{S} 1235 \mathrm{R}^{20}$ & 7T/9T & None available \\
\hline 5 & Male & Caucasian & $\begin{array}{c}\text { Family history of } \\
\text { CF mutation }\end{array}$ & $\mathrm{I} 148 \mathrm{~T}^{a} / \Delta \mathrm{F} 508$ & 9T/9T & $\begin{array}{l}\text { Fertile male who underwent carrier screening after } \\
\text { the identification of } \Delta \text { F508 in the heterozygous } \\
\text { form in his child during newborn screening, } \\
\text { child's mother is negative for } 25 \text { mutation } \\
\text { ACOG/ACMG CF panel }\end{array}$ \\
\hline 6 & Female & Armenian & $\begin{array}{c}\text { Family history of } \\
\text { CF mutation }\end{array}$ & $\begin{array}{l}\text { D110H/I148T } \\
\text { (3199del6 } \\
\text { positive) }\end{array}$ & Not determined & $\begin{array}{l}\text { Clinical information on this individual is not } \\
\text { available, despite multiple attempts to obtain. } \\
\text { DNA testing on her son revealed } \\
\text { I148T/3199del6 }\end{array}$ \\
\hline 7 & Not provided & Caucasian & Affected with CF & $\begin{array}{l}\text { V520F/I148T } \\
\text { (3199del6 } \\
\text { positive) }\end{array}$ & Not determined & None available \\
\hline 8 & Prenatal test & Middle Eastern & $\begin{array}{l}\text { Fetal echogenic } \\
\text { bowel }\end{array}$ & $\mathrm{I} 148 \mathrm{~T}^{a}$ carrier & Not determined & $\begin{array}{l}\text { Healthy male reported at age } 2 \text { years. A } \\
\text { subsequent pregnancy of this couple was } \\
\text { diagnosed with } \Delta \text { F508/I148T (before the } \\
\text { availability of } 3199 \text { del } 6 \text { reflex testing) and was } \\
\text { terminated. }\end{array}$ \\
\hline 9 & Prenatal test & Asian & $\begin{array}{l}\text { Fetal echogenic } \\
\text { bowel }\end{array}$ & $\mathrm{I}^{148 \mathrm{~T}^{a}} / \mathrm{M} 82 \mathrm{I}$ & Not determined & $\begin{array}{l}\text { Healthy female last evaluated at age } 28 \text { months } \\
\text { with no signs or symptoms of CF. Sweat } \\
\text { chloride in normal range }(11 \mathrm{mEq} / \mathrm{L})\end{array}$ \\
\hline
\end{tabular}

a3199del6 negative.

related I148T heterozygotes and $1 \mathrm{I} 148 \mathrm{~T}$ homozygote (total of 663 persons or 664 I148T chromosomes). Overall, we identified 6 unrelated individuals positive for both I148T and 3199 del6 (0.9\%). With the exception of Case 6 (Table 2), the phase of the I148T/3199del6 was not determined; however, they are presumed in-cis based on previous haplotype studies. ${ }^{9}$ These studies demonstrated that I148T occurs on a 7T or 9T background, whereas the I148T/3199del6 occurs on a 9T background. Due to ACOG/ACMG recommendations that polyT analysis only be performed as a reflex test for $\mathrm{R} 117 \mathrm{H}$-positive individuals, the polyT status of most of the patients included in this report was not determined. Excluding 15 cases referred for CF DNA testing because of a known or suspected diagnosis of CF (including fetal echogenic bowel and male infertility) or a positive family history, $0.6 \%$ of I1 $48 \mathrm{~T}$ carriers were positive for 3199del6.

Interesting cases identified are presented in Table 2. An unaffected I148T homozygote (Case 1) was detected during routine prenatal screening. Three males (cases 2-4) with congenital bilateral absence of the vas deferens (CBAVD) (I148T/ $\Delta$ F508), obstructive azoospermia (I148T carrier), and infertility (I148T/S1235R) were identified, the latter 2 negative for $5 \mathrm{~T}$ at the intron 8 polyT locus. We cannot exclude the possibility that finding I148T in the heterozygous state in a male with obstructive azoospermia (Case 3 ) is merely a coincidence. Two adults underwent $\mathrm{CF}$ carrier testing due to a known family history of a CF mutation. Case 5 was a fertile male, compound heterozygous for I148T and $\Delta \mathrm{F} 508$, who had a child identified by newborn screening as heterozygous for $\Delta \mathrm{F} 508$. His wife was negative for the ACMG CF screening panel. Case 6, a female for whom no clinical information is available, was compound heterozygous for $\mathrm{D} 110 \mathrm{H}$ and I148T/ 3199del6. Her son was noted to be positive for I148T/3199del6, confirming the haplotype in this family (assuming that his father is not a carrier for I148T or 3199del6). Case $7 \mathrm{had}$ a clinical diagnosis of CF and was positive for V520F, a CF mutation associated with pancreatic insufficiency, and I148T/3199del6.

Two fetuses with I148T and echogenic bowel were identified, both before the availability of 3199del6 reflex testing for I148T carriers. The parents of Case 8 presented to the Genetics Clinic at 12 weeks gestation for CVS because (1) the mother was a known balanced translocation carrier and (2) advanced maternal age. Chromosome analysis revealed a male karyotype with a balanced form of the maternal translocation. Fetal echogenic bowel was subsequently noted during an ultrasound performed at 18 gestational weeks. Parental CF DNA testing identified one parent as a carrier for $\Delta \mathrm{F} 508$, and the other had an unusual heteroduplex pattern in exon 4 , which was later identified as I148T (before the availability of 3199del6 reflex testing). TORCH titers were negative. The echogenic bowel had resolved on a follow-up fetal ultrasound at 21 weeks gestation. DNA testing performed after delivery was positive for I148T, but negative for $\Delta \mathrm{F} 508$. At age 2, this child was reportedly healthy with no symptoms of CF. CF testing for a subsequent 
pregnancy, performed by CVS was positive for $\Delta \mathrm{F} 508$ and I148T (again, prior to the availability of 3199del6 reflex testing). The parents were counseled regarding the inability to prenatally predict the course of symptoms and severity of CF, though $\Delta$ F508 and I148T were typically associated with pancreatic insufficient (PI) CF. The couple's 2 other children, then underwent CF DNA testing, and neither were compound heterozygous for the 2 mutations. After considering their options, the couple chose to terminate the pregnancy. In 2003, 3199del6 reflex testing was performed, and the I148T carrier parent was negative for 3199del6. The clinical geneticist responsible for this case was contacted regarding these results, so that the family could be counseled regarding these new findings.

The parents of Case 9 underwent CF carrier testing after the diagnosis of fetal echogenic bowel on ultrasound at 18 weeks gestation. One parent was positive for I148T. A previously unreported variant of unknown clinical significance, M82I $($ ATG $\rightarrow$ ATA), was identified in the other parent. M82I was incidentally detected by heteroduplex analysis of exon 3, which was used by the laboratory to screen for known CF mutations. The couple was counseled by a board-certified clinical geneticist regarding the difficulties in predicting the affect of a novel sequence change in the CFTR gene in the absence of functional studies. It was explained to the couple that M82I was most likely a benign polymorphism because codon 82 is not located in a critical region of the CFTR gene and that the substitution of methionine with isoleucine (both hydrophobic amino acids) in this region of the gene was not likely deleterious (J. Zelienski, personal communication, 2004). However, due to the presence of fetal echogenic bowel, one parent a carrier of I148T (classified as a CF mutation at that time), and the other parent a carrier of a variant of unknown clinical significance, prenatal CF testing was performed by amniocentesis. Chromosome analysis revealed a normal female karyotype. CF testing was positive for I148T and M82I. The couple was counseled regarding the possible outcomes including a normal asymptomatic child, atypical CF or mild CF, or CF with pancreatic insufficiency (due to the presence of echogenic bowel). The couple elected to continue the pregnancy. The child passed meconium within 24 hours of birth and stools were normal in the newborn period. The child was evaluated in the genetics clinic at the ages of one month and again at age 21 months. She is healthy and growing appropriately. She has no respiratory or gastrointestinal problems. A sweat chloride test performed in a CF clinic was normal (11 mEq/L). Reflex testing for 3199del6, recently performed, was negative, and the family was counseled regarding these findings.

\section{DISCUSSION}

I148T results from a T to $\mathrm{C}$ substitution at nucleotide 575 in exon 4 of the CFTR gene. This sequence change was initially reported to the CF Consortium in $1990 .{ }^{1}$ I148T is located in the first cytoplasmic loop of the first membrane spanning domain of CFTR ${ }^{14}$ and results in the substitution of a hydrophobic amino acid, isoleucine, for a polar amino acid, threonine. This region is conserved in humans, mouse, and bovine. ${ }^{15}$ Despite the fact that functional studies revealed normal processing, gating, and conductance of the CFTR, ${ }^{14} \mathrm{I} 148 \mathrm{~T}$ was considered to be a severe CF mutation due to its presence in patients with classic CF and a second pathological CF mutation. ${ }^{4,5,7}$

3199del6, a deletion of ATAGTG from nucleotide 3199, is located in CFTR exon 17a and results in the deletion of isoleucine and valine at codons 1023 to 1024 within the second membranespanning domain. 3199del6 was reported to the CF Consortium in 1998 in a pancreatic insufficient CF patient with I148T and $3199 \mathrm{del} 6$ on the same chromosome and $\Delta \mathrm{F} 508$ on the other chromosome. ${ }^{1}$ Another study noted a patient with severe CF and 3199del6 and I148T, though it is not noted specifically whether these mutations are in-cis or in-trans. ${ }^{16} 3199$ del6 also occurs in the absence of I148T, as a patient with severe CF has been reported with 3199del6 (negative for I148T) and G542X.17

Recent data suggests that 3199 del6 is the deleterious mutation among I148T/3199del6 complex alleles. ${ }^{9}$ It is puzzling that early reports of patients with classic CF and I $148 \mathrm{~T} / \Delta \mathrm{F} 508 \mathrm{did}$ not identify 3199del6, despite the fact that 2 reports described performing mutation analysis of the entire CFTR coding region and splice site junctions either by a screening method (DDGE) or DNA sequencing. 5,7 However, a recent report identified 3199del6 in 24 French-Canadian CF patients originally described as compound heterozygous for I148T and a severe CF mutation. ${ }^{11}$ Though there is little doubt that 3199del6 is a deleterious CF mutation, the clinical significance of I148T in the absence of 3199del6 is unclear, as we have identified this genotype in 3 males with infertility, two of the obstructive type.

In 2001, I148T was included in the ACMG/ACOG CF panel due to its high incidence in the general population. ${ }^{2,3}$ However, recent data revealed that I $148 \mathrm{~T}$ accounts for $\approx 0.06 \%$ of $\mathrm{CF}$ chromosomes, ${ }^{9}$ less than the $0.1 \%$ frequency for inclusion in the CF screening panel. Therefore, based on its low frequency among CF patients and questions concerning the phenotype associated with I148T, its inclusion in the CF screening panel should be reconsidered. 3199del6 is not among the most common reported CF mutations in the world and is not routinely screened for in most diagnostic laboratories. However, as this and other studies ${ }^{9,12}$ have identified this mutation at a low frequency among I148Tcarriers, the addition of 3199del6 to the CF panel would not significantly increase the detection rate of the panethnic CF screening panel.

In our collaborative study of 663 I $148 \mathrm{~T}$ carriers, $0.9 \%$ also had 3199del6. Excluding subjects tested because of a suspected or clinical diagnosis of CF or positive family history, the frequency of 3199del6 decreased to $0.6 \%$. We identified 7 unrelated individuals with I148T and a second CF variant, 2 of whom also carried 3199del6. One patient with a known CF severe mutation, V520F, and I148T(3199del6) had a clinical diagnosis of $\mathrm{CF}$, although no specific clinical information is available. The second individual underwent testing due to the presence of two different CF mutations in her two children and was found to have the genotype D110H/I148T(3199del6). No clinical information on this individual was available to the lab- 
oratory performing the testing. One of her sons was subsequently found positive for I148T/3199del6. D110H is a rare CF sequence variant, originally reported in a mildly affected CF patient, ${ }^{18}$ and recently in homozygous form in an infant with metabolic alkalosis. ${ }^{19}$

The remaining I148T compound heterozygotes were negative for 3199del6. Among these include a fertile male with the genotype I148T/ $\Delta \mathrm{F} 508$ who underwent carrier screening due to the presence of a $\Delta \mathrm{F} 508$ in his child identified by newborn screening and an asymptomatic female, homozygous for I148T, identified by routine CF prenatal carrier screening. Three males with infertility were I148T positive. One with obstructive azoospermia was heterozygous for I148T, but no other mutation (including 5T) was detected. A male with unspecified infertility was compound heterozygous for I148T and S1235R $(7 \mathrm{~T} / 9 \mathrm{~T})^{20}$ and a male with CBAVD was compound heterozygous for I148T and $\Delta$ F508 (polyT status not determined).

Two fetuses with echogenic bowel and I148T were identified, both before the availability of 3199del6 reflex testing; however, both I148T carrier parents were subsequently shown to be negative for 3199del6. One fetus was compound heterozygous for I148T and M82I, a previously unreported variant of unknown clinical significance. After extensive genetic counseling the pregnancy was continued and the child is healthy, with no signs of $\mathrm{CF}$ and a normal sweat chloride test. The parents of the other case underwent CF carrier screening after the diagnosis of fetal echogenic bowel in a pregnancy. One parent was identified as a $\Delta \mathrm{F} 508$ heterozygote and the other with an unknown sequence change in exon 4 , later identified as I148T. Their child was positive for I148T only and at age 2 was reported as healthy. This couple underwent prenatal CF testing for a subsequent pregnancy, and the fetus was identified as positive for $\Delta \mathrm{F} 508$ and I148T. After genetic counseling, which included the inability to prenatally predict the course of symptoms and severity of CF, although $\Delta \mathrm{F} 508$ and I148T were typically associated with PI CF, the couple chose to terminate the pregnancy. The I148T carrier parent in this relationship is now known to be negative for 3199del6.

Our data supports previous findings that a small number of individuals with I148T are positive for 3199del6. The identification of 3 males with infertility and I148T ( 2 of the obstructive type and 2 who are compound heterozygous for I148Tand another CF mutation) cannot exclude the possibility that I148T alone may be associated with atypical or mild CF. Therefore, care must be taken when discussing genotype-phenotype correlations, especially during prenatal diagnosis. Unfortunately, we were unable to obtain detailed information for all patients in whom I148T was identified; therefore, we cannot make reliable predictions on the phenotype of I148T alone. Reflex testing for 3199del6 should be considered whenever I148T is identified. Such testing is of particular importance in any patient with features of CF or whenever one member of a couple carries a deleterious $\mathrm{CF}$ mutation and the other member carries I148T. Further studies are necessary to determine if I148T, in the absence of 3199del6, is associated with mild or atypical CF, including male infertility.
In summary, these data suggest that $\mathrm{I} 148 \mathrm{~T}$ is not an appropriate mutation for CF screening in the general population. Furthermore, the frequency of 3199del6 is $<0.1 \%$, which is below the frequency criteria for inclusion in the ACMG/ACOG CF mutation screening panel. We recommend that I148T be deleted from the CF screening panel and that 3199del6 not be added. However, if it is confirmed that 3199del6 accounts for a significant proportion of CF alleles in the French-Canadian population, laboratories may wish to offer analysis for this mutation specifically to members of this ethnic group.

\section{References}

1. Hospital for Sick Children. Toronto, Cystic Fibrosis Mutation Database. Available at: http://www.genet.sickkids.on.ca/cftr/ Accessed February 2004.

2. Grody WW, Cutting GR, Klinger KW, Richards CS, Watson MS, Desnick RJ. Laboratory guidelines for population-based CF carrier screening. Genet Med 2001;3: $456-461$.

3. American College of Obstetrics and Gynecology and American College of Medical Genetics. Preconception and prenatal carrier screening for cystic fibrosis. Clinical and laboratory guidelines. Washington, DC. American College of Obstetrics and Gynecology publication, 2001.

4. Kristidis P, Bozon D, Corey M, Markiewicz D, Rommens J, Tsui L-C et al. Genetic determination of exocrine pancreatic function in cystic fibrosis. Am J Hum Genet 1992;50:1178-1184.

5. Bozon D, Zielenski J, Rininsland, Tsui L-C. Identification of four new mutations in the CFTR gene: I148T, L1077P, Y1092X and 2183AA-G. Hum Mut 1994;3:330-332.

6. Castaldo G, Rippa E, Raia V, Salvatore D, Massa C, deRitis G et al. Clinical features of CF patients with rare genotypes. J Med Genet 1996;33:73-76.

7. DeBraekeleer M, Mari C, Verlingue C, Allard C, LeBlanc JP, Simard F et al. Clinical features of cystic fibrosis patients with rare genotypes in Saguenay Lac-Saint-Jean (Quebec, Canada). Ann Genet 1997;40:205-208.

8. The Cystic Fibrosis Genetic Analysis Consortium. Population variation of common cystic fibrosis mutations. Hum Mut 1994;4:167-177.

9. Rohlfs EM, Zhou Z, Sugarman EA, Heim RA, Pace RG, Knowles MR et al. The I148T CFTR allele occurs on multiple haplotypes: a complex allele is associated with cystic fibrosis. Genet Med 2002;4:319-323.

10. Strom CM, Huang D, Buller A, Redman J, Crossley B, Anderson B et al. Cystic fibrosis screening using the College panel: Platform comparison and lessons learned from the first 20,000 samples. Genet Med 2002;4:289-296.

11. Ruchon AF, Ryan SR, Rozen R, Scott P. Genotype-phenotype correlation between the complex allele I148T-3199del6 and cystic fibrosis. Am J Hum Genet 2003;73:A1402.

12. Buller A, Olson S, Redman J, Hantash F, Chen R, Strom CM et al. Brief report: frequency of the cystic fibrosis 3199del6 mutation in individuals heterozygous for I148T. Genet Med 2004;6:108-109.

13. Strom CM, Huang D, Chen C, Buller A, Peng M, Quan F et al. Extensive sequencing of the cystic fibrosis transmembrane regulator gene: assay validation and unexpected benefits of developing a comprehensive test. Genet Med 2003;5:9-14.

14. Seibert FS, Jia Y, Mathews CJ, Hanrahan JW, Riordan JR, Loo TW et al. Diseaseassociated mutations in the cytoplasmic loops 1 and 2 of cystic fibrosis transmembrane conductance regulator impede processing or opening of the channel. Biochemistry 1997;36:11966-11974.

15. Diamond G, Scanlin TF, Zasloff MA, Bevins CL. A cross-species analysis of the CFTR. J Biol Chem 1991;266:22761-22769.

16. Wong L-J, Wang J, Zhang X-H, Hsu E, Heim RA, Bowman CM et al. Improved detection of CFTR mutations in southern California Hispanic CF patients. Hum Mut 2001;18:296-307.

17. Buyse IM, McCarthy SE, Pace RP, Schmitt ES, Ward P, Oermann C et al. Diagnostic testing for cystic fibrosis by MALDI-TOF mass spectrometry: identification of a CF patient with a G542X/3199del6 genotype. Am J Hum Genet 2003;73:A1401.

18. Dean M, White MB, Amos J, Gerrard B, Stewart C, Khaw K-T et al. Multiple mutations in highly conserved residues are found in mildly affected cystic fibrosis patients. Cell 1990;61:863-870.

19. Weller F, Wiebicke W, Tummler B. [Turkish infant with hypoelectrolytemia and metabolic alkalosis as the sole manifestations of a mild form of cystic fibrosis (mutation D110H)]. Klin Padiatr 2000;212:41-43.

20. Monaghan KG, Feldman GL, Barbarotto GM, Manji S, Desai TK et al. Frequency and clinical significance of the S1235R mutation in the cystic fibrosis transmembrane conductance regulator gene: results from a collaborative study. Am J Med Genet 2000;95:361-365. 